$10.1590 / \mathrm{S} 1414-40772015000300012$

\title{
La formación educativa socio-jurídica para estudiantes universitarios
}

\author{
Xiomara Cabrera Cabrera \\ Raquel Diéguez Batista
}

Resumen: Se propone como aporte teórico un modelo para la dinámica formativa socio-jurídica de los estudiantes universitarios, desde la formación axiológica- jurídica, que contribuya al perfeccionamiento de la dinámica de este proceso educativo, lo que redunda en mejores comportamientos éticos profesionales y ciudadanos en los estudiantes universitarios, coherentes con las exigencias de la sociedad actual en un nuevo contexto de cambios en lo social, económico y jurídico. Estos resultados forman parte de una investigación realizada en la Universidad "Máximo Gómez Báez" de Ciego de Ávila, Cuba en el periodo comprendido entre el año 2011 y 2013, utilizando métodos y técnicas de investigación científicas, corroborados en diferentes carreras de esta universidad.

Palabras clave: Cultura integral. Formación axiológica-jurídica. Entorno socio-legal. Aprehensión identitaria.

\section{A formação educativa sociojurídica para estudantes universitários}

Resumo: Um modelo para a dinâmica de formação sóciojurídica de estudantes universitários de formação axiológico-jurídica, que contribua para a melhoria da dinâmica desse processo educacional, resultando em um comportamento ético profissional e cidadão é proposto como uma contribuição teórica, coerente com as exigências da sociedade moderna em um novo contexto de mudanças sociais, económicas e jurídicas. Estes resultados são parte de uma investigação conduzida na Universidade "Maximo Gomez Baez" de Ciego de Avila, Cuba, no período entre 2011 e 2013, usando métodos e técnicas de pesquisa científica, corroboradas em várias carreiras desta universidade.

Palavras-chave: Cultura Integral. Formação axiológico-jurídica. Ambiente sócio-legal. Apreensão de identidade.

\section{The socio-legal educational training for college students}

Abstract: Theoretical contribution is proposed as a model for the dynamics of socio-legal training of college students from axiological-legal training, which contributes to the improvement of the dynamics of this educational process, resulting in better professional and ethical behavior in public college students, consistent with the demands of modern society in a new context of changes in the social, economic and legal realms. These results are part of an investigation conducted at the University "Maximo Gomez Baez" Ciego de Avila, Cuba in the period between 2011 and 2013, using methods and techniques of scientific research, corroborated in several academic programs at this university.

Key words: Integral culture. Axiological-legal Training. Socio-legal environment. Identity Apprehension. 


\section{Introducción}

Uno de los retos más significativos de hoy día es la formación de los profesionales para enfrentar la construcción y desarrollo de la cultura, en la diversidad y complejidad de los conocimientos y métodos generados sobre los múltiples procesos naturales, sociales y del pensamiento, en un mundo que se transforma a ritmos nunca antes alcanzados. Tal situación requiere de cambios profundos en las concepciones formativas, las cuales han de corresponderse con el alcance de las transformaciones y necesidades culturales, sociales y profesionales.

Los estudiantes universitarios en su proceso de formación educativa sociojurídica, requieren del perfeccionamiento de una cultura integral, la cual permita ampliar las capacidades, para que desarrollen una capacidad transformadora profesionalizante, con una alta competencia en el desempeño científico, social y jurídico, así como tener un elevado compromiso y responsabilidad social con la patria. El profesional universitario por las disimiles funciones y roles que cumple en la sociedad actual, comprometido con su formación, necesita tener conocimiento del quehacer y la practica social y jurídica del país.

Sin embargo en la actualidad, aún existen, insuficientes referentes teóricos y metodológicos que abordan el estudio del proceso formativo social y jurídico de los estudiantes universitarios desde una dinámica que conduzca a un tratamiento didáctico-metodológico, que potencie la capacidad transformadora como esencia para una actuación más comprometida y responsable socialmente, a partir del fortalecimiento de la cultura ética-jurídica.

Un análisis del contenido, exige evidencia de una diversidad de posturas convergentes en las cuales subyace la necesidad de instrumentar una aproximación teórica que posibilite la identificación, aprehensión, generalización y la sistematización de dicho proceso formativo, razón por la cual, el objetivo del presente trabajo es proponer un modelo de la dinámica del proceso de formación socio-jurídica para estudiantes universitarios.

\section{Sección de Materiales y Métodos}

En la investigación realizada en la Universidad “Máximo Gómez Báez” de Ciego de Ávila, en el periodo comprendido en el curso académico 2011-2012 y 2012-2013, se emplearon los siguientes métodos y técnicas de investigación científica en su realización: 
- Método Histórico Lógico para la determinación de las tendencias históricas del proceso de formación socio-jurídica en los estudiantes universitarios y su dinámica.

- Método de Análisis y Síntesis para todo el estudio realizado del proceso de formación socio-jurídica en los estudiantes universitarios, transitando toda la lógica de investigación científica.

- Método Holístico Dialéctico para modelar la dinámica del proceso de formación socio- jurídica en los estudiantes universitarios, debiendo concebir el objeto y el campo a través de las relaciones dialécticas que se establecen.

- Método Sistémico Estructural Funcional para diseñar la estrategia socio- jurídico en los estudiantes universitarios y su dinámica.

- Métodos Estadísticos: Pruebas de Hipótesis para constatar la significación de la transformación producida al aplicarse la estrategia propuesta.

- Encuestas, entrevistas, observación y taller de socialización, para el diagnóstico del estado inicial del objeto y campo de la investigación.

- Criterio de expertos para valorar la factibilidad y pertinencia científica científico metodológica del modelo.

\section{Resultados}

"La investigación retoma los postulados de la Pedagogía de la Educación Superior, desde la Concepción Científica de lo Holístico Configuracional" (FUENTES, 2009, p. 8), como aporte teórico metodológico y método científico general para la construcción del modelo, los principios del carácter formativo del contexto, y la sistematización formativa; desde la reflexión que permite la sucesión de movimientos como expresión de las configuraciones y dimensiones, por las que transita la dinámica para la formación socio-jurídica en los estudiantes universitarios.

"El reconocimiento de la carencia de valores éticos profesionales en la sociedad contemporánea" (FABELO, 2011, p. 24), trasciende a los estudiantes universitarios, justifica la estructuración del modelo y el camino hacia la construcción de la práctica socio-jurídica sistematizada en la trasformación del estudiante para el desempeño profesional, como parte de la formación permanente y para toda la vida, que tiene en cuenta un conjunto de relaciones que se establecen entre las actividades docentes curriculares, extracurriculares, 
de extensión universitaria para un actuar ético profesional con conocimientos de las regulaciones legales de su profesión y la sociedad.

Para las autoras Cabrera y Diéguez (2013, p. 65), "El proceso de formación socio-jurídico de los estudiantes universitarios se identifica con la sucesión de procesos internos formativos que se encaminan a la profundización de la ética profesional y la cultura jurídica".

En tal sentido, el proceso tiene un carácter renovador y transformador, expresado en la sistematización de la responsabilidad socio-jurídica en el entorno universitario, que está en constante transformación de conocimientos, de experiencias y saberes culturales; permite a partir de la responsabilidad como valor, buscar compromisos colectivos para un mejor desempeño en la sociedad, orienta y conduce la forma de actuar en el plano de lo ético y la moral; espacio para lograr la socialización e intercambio de prácticas sociales (CAPDET, 2011).

En esa misma medida, el proceso de formación socio-jurídico posee el encargo de una nueva cultura axiológica jurídica fomentada en valores éticos y sociales para consolidar la formación integral del estudiante hacia el compromiso social, la conservación y respeto hacia la diversidad cultural ética y jurídica.

En la medida en que el estudiante logra la aprehensión axiológica en interrelación con la responsabilidad adquirida en el entorno universitario, desde la diversidad contextual, hace juicios, valoraciones (ESPINOSA, 2008), lo que contribuye a profundizar la visión sobre su actuación en la sociedad, lo que posibilita un estado deseado del saber social desde una mirada diferente, potencia la apropiación de la cultura ética reglamentaria.

En tal sentido, se considera un proceso complejo, examinado a través de los etapas del proceso de formación permanente, partiendo de la mejora y el perfeccionamiento profesional que se constituye en la práctica de la ética profesional y la cultura jurídica, a la vez de significar el contexto universitario donde se materializan los comportamientos sociales de los estudiantes durante su formación.

Se ha tenido en cuenta, la Concepción Científica Holístico Configuracional de Fuentes (2009), que desde lo epistemológico y metodológico, posibilita fundamentar la orientación intencional del sujeto, a partir de las relaciones dialécticas que acontecen, piedra angular en la interpretación y caracterización de los procesos sociales, que se desarrollan a través de una práctica didáctica interactiva.

De ahí, que en la investigación para fundamentar el proceso formativo se establecen procesos, dentro de ellos, la identificación del entorno socio-jurídico 
de la comunidad universitaria y la aprehensión de la cultura identitaria ética-jurídica profesional, categorías que en la dinámica del proceso de formación socio-jurídica de los estudiantes universitarios se dan como una relación dialéctica (BELLO, 2010). Individualización que realiza el sujeto del medio donde ejecuta sus principales actividades sociales, enmarcadas estas dentro de un ámbito legal, haciendo una valoración social de su entorno como expresión de los conocimientos, en interacción recíproca con otros sujetos, expresada en los momentos objetivos y subjetivos del devenir de la vida intra y extra universitaria, esto lo motiva a desarrollar su aprendizaje con respeto a la ética profesional y las normativas legales vigentes, surgiendo así, en el sujeto un sentimiento de pertenencia por su comunidad universitaria y su patria.

En tanto, en la aprehensión de la cultura identitaria ética-jurídica profesional el sujeto debe proyectarse en la asimilación inmediata de ideas, conocimientos y valoraciones para la protección del entorno socio-jurídico universitario y en defensa de su identidad estudiantil que requiere de un proceso individual y colectivo para reflexionar acerca de las experiencias y saberes (BARRIOS, 2009), lo que permite ejercitar su autonomía personal e intelectual, describir sus experiencias, descubrir entre todos, lo que le es común y lo que los diferencia, lo que esperan de su actuación y lo que hacen para el cumplimiento de tareas de impacto social como resultado de un conjunto de interacciones múltiples con el contexto de aprendizaje, la propia universidad, y la comunidad.

La cultura jurídica se refiere al conocimiento externo del sistema legal y que el estudiante universitario incorpora como ciudadano, visto como una obligación, una controversia, un mandato al cumplimiento de una norma escrita y que en la medida en que haya espacio entre sus miembros se conoce mejor y evita su infracción. Es la lucha contra manifestaciones negativas de corrupción, elementos importantes que una comunidad utiliza para entender y aplicar sus normas y regulaciones.

En tal sentido, la aprehensión de la cultura identitaria ética-jurídica profesional, es un proceso de interpretación del derecho, de caracterización de los valores éticos y de ampliación de la cultura jurídica, a partir de que, la universidad, es una institución forjadora de identidades, de actitudes y comportamientos éticas, al mismo tiempo, forma valores, que los socializa para transformarlo en un ser social, integrante de una comunidad universitaria, haciéndolo participe de los nuevos cambios que acomete la sociedad cubana actual.

La categoría generalización de la cultura axiológica- jurídica profesional, implica difusión y divulgación de mecanismos que permitan a los sujetos prestar atención al mejoramiento de las cualidades humanas, reglas de disciplina y 
convivencia universitaria, actuaciones ante determinadas situaciones, lo que permite un equilibrio de sus fortalezas, debilidades y oportunidades, pero también sus retos futuros, y con ello reorientar los valores éticos ciudadanos y jurídicos para una formación de calidad y pertinencia.

Supone formar al sujeto para prevenir un actuar irresponsable y conductas no acorde con las normas reglamentadas, promover la comunicación y el acercamiento entre los sujetos, la comprensión mutua, el diálogo ameno sobre los problemas que hoy se suscitan en la sociedad y la interacción reflexiva de la práctica, por tanto, es el proceso que implica intencionalidad para la transformación de nuevos conocimientos, capaces de crear habilidades, estrategias cívicas, valores y prácticas socio-jurídicas, que incrementen su ética profesional y su cultura jurídica, según las necesidades de las carreras y las expectativas de estas en la sociedad como profesional formado, requiriendo para ello de un proceso de formación sistemático, coherente e intencionado.

El movimiento establecido entre los procesos ya enunciados, garantizan un primer momento de fortalecimiento en la formación socio-jurídica del estudiante universitario, como nivel cualitativamente superior de interpretación de la sistematización de la práctica socio-jurídica profesional, que se concreta en la dimensión integradora jurídica-axiológica, la cual emerge como una configuración de orden superior a partir de la relación dialéctica que se produce entre la identificación del entorno socio-jurídico de la comunidad universitaria, la aprehensión de la cultura identitaria ética-jurídica profesional, y la generalización de la cultura axiológica- jurídica profesional, se sitúa como síntesis dinamizadora de dicha contradicción la sistematización de la praxis socio-jurídica profesional.

La dimensión integradora jurídica-axiológica, emerge como expresión del movimiento de un proceso que permite incorporar a los estudiantes universitarios en su formación socio-jurídica permanente, para la apropiación de la cultura ética-legal de manera crítica, profunda, flexible e integradora, sobre bases científicas, que le permita llegar a conclusiones, deducciones y proponer alternativas ante la solución de problemas en un campo específico de la realidad social; como premisas necesarias para la formación cultural axiológica y jurídica.

Esta dimensión, a su vez, fortalece el papel formativo de los estudiantes universitarios como futuros profesionales, capaces de cumplir compromisos sociales, permeadas de valores pedagógicos y jurídicos que le permitan convivir en la sociedad, expresa el carácter integrador que se revela en procesos de desarrollo, de cualquier profesional, donde no es posible comprender las 
situaciones a las que la praxis profesional le exige enfrentar, si no se conoce el sistema de valores éticos existentes, el dominio de instrumentos pedagógicos precisos, la apropiación de conocimientos básicos sobre las normas morales, de derecho y otras disciplinas científicas afines con cada carrera para ejercer la profesión desde la perspectiva de un profesional con convicciones profundamente revolucionarias humanistas y de justicia social.

Por lo que, la dimensión integradora jurídica-axiológica, es el proceso de la sistematización en la práctica del estudiante universitario, que permite una mayor profundización de los valores éticos-jurídicos para alcanzar una cultura integral sobre la preparación y el entrenamiento diario y sistemático en la brigada, colectivo pedagógico, la carrera y otros espacios intra y extra universitarios.

Par validar los resultados se emplean de manera interrelacionada métodos empíricos y técnicas de investigación: Se tuvo en cuenta el criterio de 27 expertos, vinculados a la formación educativa en la educación superior, dentro de ellos 12 doctores, 11 magister y 4 especialistas con categorías de auxiliar y titular. Existiendo concordancia entre los expertos, para lo cual se aplicó la prueba de Kendal y el cálculo del Coeficiente de Alfa de Cronbach para la confiabilidad de los instrumentos aplicados. Coincidiendo los expertos en la necesidad del perfeccionamiento de la formación socio-jurídica para estudiantes de la universidad de Ciego de Ávila.

Los resultados se ejemplifican en la carrera de Ingeniería Civil del Curso Regular Diurno, modalidad presencial. Se trabajó con el colectivo de profesores, responsabilizados con el proceso formativo, a los cuales se les preparó desde las reuniones de colectivo pedagógico en temáticas axiológicas, ética de la profesión y contenidos reglamentarios legales, en las que se le impartieron las incidencias de las políticas educativas del Ministerio de Educación Superior respecto al reglamento disciplinario para estudiantes, además en los temas contemplados en la estrategia cívica y de preparación jurídica, como parte del proceso de desarrollo de la cultura integral en la institución universitaria.

Se alcanzan logros y transformaciones en el actuar de los estudiantes universitarios que fueron corroborados con la aplicación de una prueba de hipótesis de los rangos con signo de Wilcoxon, al modificar su conducta desde el actuar ético, relacionándolos con su profesión, aunque no puede hablarse de una transformación radical ya que las acciones aún continúan aplicándose, sin embargo, puede decirse que aprendieron nuevas formas de trabajo en grupo, compartiendo en los espacios de la brigada y la universidad de una forma interactiva y reflexiva, que contribuyeron a desarrollar otras aptitudes interac- 
tivas para la toma de decisiones y problematizar la realidad en la búsqueda de soluciones a dilemas y conflictos que ocurren en la vida universitaria y fuera de este entorno.

\section{Conclusiones}

El modelo teórico propuesto, además de su relevancia, posee una estructuración coherente y lógica en las relaciones que se establecen entre sus procesos categoriales, así como fundamentos teóricos coherentes, a la vez que, resulta novedoso en relación a la problemática investigada, criterio que responde a las exigencias de una formación educativa socio-jurídica para estudiantes universitarios.

En la práctica se aplicaron métodos empíricos de investigación, a partir del cual, es posible explicar la dinámica de este proceso formativo desde la sistematización de la práctica socio-jurídica profesional, lo que permitió corroborar las transformaciones alcanzadas en los estudiantes.

Los estudiantes universitarios mejoraron su disposición hacia el cumplimiento de tareas de impacto de su entorno, lograron transformar problemas concretos al apropiarse de contenidos en temas legales, enriqueciendo a la vez su formación para un desempeño pertinente y responsable socialmente.

\section{Referencias}

BELLO, L. La ética como filosofía primera, una fundamentación desde la ética de la responsabilidad en el cuidado médico. Cuadernos de Bioética, La Habana, v. 21, n.1, p. 13-20, 2010.

\section{BARRIOS, F. Modelo de gestión formativa institucional de formación} profesional permanente de los docentes. 2009. Tesis (Doctor en Ciencias Pedagógicas) - Centro de Estudios Manuel F. Gran, Santiago de Cuba, 2009.

CABRERA, X.; DIÉGUEZ, R. Orientaciones metodológicas para el desarrollo del proceso de formación socio-jurídica en los estudiantes universitarios. Revista Multiciencias, Venezuela, v. 13, n. 1, p. 61- 67, 2013.

CAPDET, K. Dinámica formativa de la cultura bioética profesional. Tesis (Doctor en Ciencias Pedagógicas) - Centro de Estudios Manuel F. Gran, Santiago de Cuba, 2011. 
ESPINOSA, J.A. Gestión de la cultura profesional en la educación superior. 2008. Tesis (Doctor en Ciencias Pedagógicas) - Instituto Superior Pegagógico "José de la Luz y Caballero", Holguín, 2008.

FABELO, J. R. Los valores y sus desafíos actuales. La Habana: Editorial José Martí, 2011.

FUENTES, H. C. Pedagogía y didáctica de la educación superior.

Material del Centro de Estudios "Manuel F. Gran". Santiago de Cuba:

Universidad de Oriente, 2009.

Xiomara Cabrera Cabrera - Universidad de Ciego de Ávila Ciego de Ávila | Cuba. Contato: xiomarac@derecho.unica.cu

Raquel Diéguez Batista - Universidad de Ciego de Ávila Ciego de Ávila | Cuba. Contato: raqueldb1961@yahoo.es

Artigo recebido em 12 de novembro de 2013 e aprovado em 8 de julho de 2014. 
